\title{
VALIDATION OF TIE-POINT CONCEPTS BY THE DEM ADJUSTMENT APPROACH OF TANDEM-X
}

\author{
M.Huber, A. Gruber, B. Wessel, M. Breunig, A. Wendleder \\ German Aerospace Center (DLR), Oberpfaffenhofen, 82234 Wessling, Germany, \\ Tel. +49 8153282895 \\ [Martin.Huber, Astrid.Gruber, Birgit.Wessel, Markus.Breunig, Anna.Wendleder]@dlr.de
}

\begin{abstract}
The aimed accuracies for the final TanDEM-X DEM of $10 \mathrm{~m}$ absolute and $2 \mathrm{~m}$ relative height error will be ensured by calibration data. One crucial data set for the relative accuracy is tie-points that connect adjacent DEM acquisitions in the approximately $4 \mathrm{~km}$-overlap-area with each other. In this paper an improved concept for tie-point candidates is presented that is based on averaging a larger region instead of comparing single points. This concept should be more robust against noise. It is validated by applying the DEM calibration on a simulated test area and if available on real TanDEM-X data. Also, the DEM calibration will be validated for the first time on a larger "real" test site by applying the TanDEM-X processing scenario.
\end{abstract}

Index Terms - TanDEM-X, Calibration, Tie-points

\section{INTRODUCTION}

On the one hand tie-points should ensure the $2 \mathrm{~m}$ relative height requirement for neighbored DEMs on the other hand each single interferometric height is distorted by Gaussian and in addition especially at the borders of the DEMs by colored noise so that the noise reaches up to $2 \mathrm{~m}$ to $5 \mathrm{~m}$. For serving as reliable tie-point on the first place the noise level has to be reduced, and on the second just reliable points have to be chosen (e.g. areas without layover, shadow, phase unwrapping errors).

Two different tie-point extraction methods are compared to each other (Section 2). Based on these tie-point sets for validation the DEM adjustment is carried out that estimates residual height offsets and deformations of the interferometric DEMs [1], [2]. As test and reference area serve a simulated test site based on SRTM data (Section 3). Also, the DEM calibration will be validated by this scenario, e.g. by varying the number of ground control points and tie-points in order to investigate the accuracy of the estimated parameters.

\section{TIE-POINT CONCEPT}

Tie-points are used as input for the DEM adjustment and therefore located in overlap areas of adjacent DEM acquisitions (data takes). They should be located at the center of the overlapping area, because the quality of each DEM reduces towards its edge due to noise. The overlap center can be calculated beforehand as the look angles of the acquisitions are already defined. This information is used to calculate the coordinates of equidistant spaced points. A $1 \times 1 \mathrm{~km}$ chip is extracted around this position as basis for delivering the actual tie-point information. In the following two tie-point extraction methods are described.

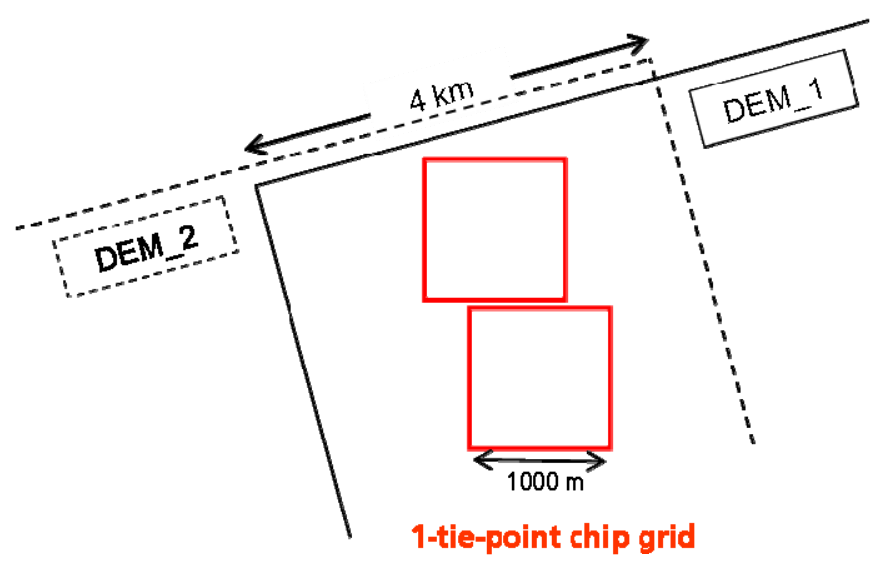

Fig. 1: Tie-point chips in overlapping centers

\subsection{Single Point Approach}

The $1 \mathrm{x} 1 \mathrm{~km}$ chip extracted from the first available DEM is used as master chip. Inside this chip the most appropriate location for the tie-point is estimated. Areas, which are flagged as shadow, layover, water or having a height discrepancy to the reference SRTM-DEM are not taken into account. A filtering window (e.g. 9 pixels) is applied to identify the most flat area, where the impact of a potential horizontal error is minimized. This position is identified as tie-point location. The corresponding height value is 
averaged over a filtering window (e.g. 3 pixels) around the tie-point to reduce the effect of noise present in the DEM.

The location of the tie-point defined by the master chip for the first time is the default position for all subsequently acquired DEMs.

\subsection{Area-base Approach}

In the area-based tie-point approach the whole $1 \times 1 \mathrm{~km}$ chip area is taken into account to define a medium difference height between two chips. Although, pixels, which are flagged as shadow, layover, water or having a height discrepancy to the reference SRTM-DEM, are not taken into account. A threshold could be applied to the coherence in addition to identify unreliable pixels. A histogram is calculated only for the area, which is 'valid' in both chips. From this, the median height value is assigned to the tiepoint instead of the mean, reducing the impact of outliers. Along with the latitude and longitude of the chip center the heights of the two corresponding chips serve as input for the adjustment. The chips need to have more than a certain percentage of valid pixels to be accepted. An indicator for the reliability of the tie-point is the standard deviation of the difference-chip. A small standard deviation would indicate similar topography at the same position.

\section{VALIDATION OF THE TIE-POINT CONCEPT}

\subsection{Simulated test site based on SRTM data}

For having real terrain data and on the same way reference data, a test site based on SRTM data was simulated. The test site extends from Winnipeg (Canada) over Minnesota and Iowa to Arkansas (see Fig. 2). It consists of 13 data takes (each divided into 10 RawDEMs) and is about $150 \mathrm{~km}$ wide and $1500 \mathrm{~km}$ long. In the north and in the south the area is quite flat, in the middle (Nebraska and Iowa) it is hilly. Most of the area is covered by cropland. Therefore it can be assumed, that the ICESat points [3] and the SRTM heights have a good quality.

For the tests the SRTM heights were distorted by a simulated error function ( $3^{\text {rd }}$ order polynomial) and additionally a random noise of $2 \mathrm{~m}$ was added. The offset of a TanDEM-X DEM is expected to be few meters, the tilts and the other coefficients are expected to be at dm-level and cm-level respectively.

\subsection{DEM calibration results}

The DEM calibration will estimate the residual height offsets and errors of the data takes according to the functional error model by a least squares adjustment using the elevation of the tie points in the interferometric DEMs of overlapping regions [1], [2]. The height offsets to
WGS84 are estimated by introducing absolute height reference data

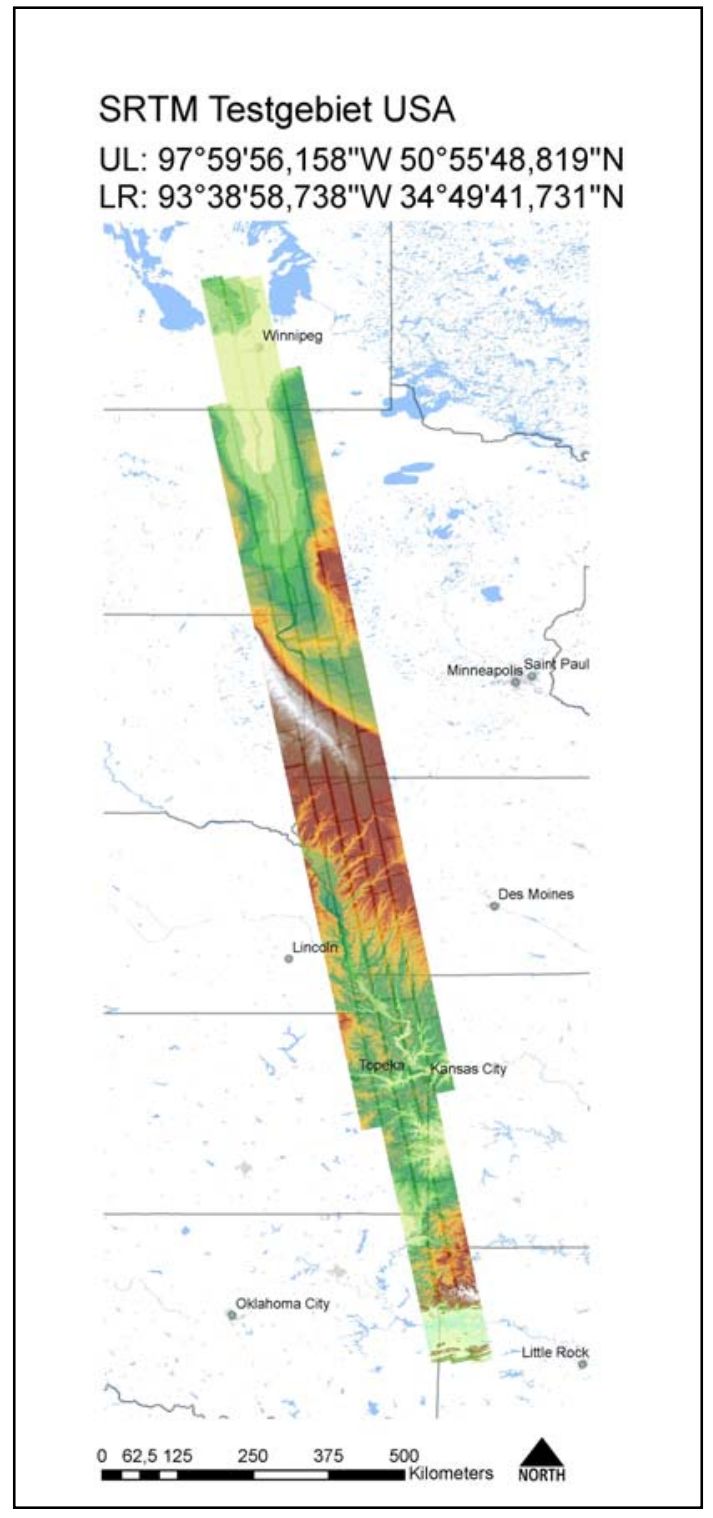

Fig. 2: SRTM test site

like ICESat data [3]. The standard deviation and the significance of the estimated unknowns act as an indicator for the quality of the results. The estimation of the coefficients is an iterative process, starting with the estimation of a polynomial function of third order and reducing the number of coefficients when the resulting significances are not high enough.

As SRTM itself does not correspond to the ICESat data in a first adjustment step the test site is referenced to ICESat data to serve as basis for comparisons. Further data for comparison are a short GPS-track and several LaserDEMs. Also, a high number of ground control points (ICESat) is available for the test site. 
Former tests with simulated data have shown, that a simulated offset can always be estimated, whereas simulated tilts can only be estimated if enough ground control points are available. The more ground control points are used the more coefficients of the polynomial function can be estimated. They have also shown that the reduction of the noise of the tie-points improves slightly the estimation of the parameters.

\section{CONCLUSION}

The advantage of the area-based approach lies in the median of several noisy heights. The median difference behaves more stable against single tie-points especially when dealing with noisy interferometric data. As the noise of the tiepoints can be reduced by averaging a larger area compared, the DEM calibration results are expected to be better with the area-based tie-point approach. If available the tests will also be carried out on mono-static TanDEM-X data of the commissioning phase.

\section{REFERENCES}

[1] Gruber, A., B. Wessel, M. Huber, 2009. TanDEM-X DEM calibration: Correction of systematic DEM errors by block adjustment. Proceedings of IGARSS 2009, Cape Town, South Africa.

[2] Wessel, B., A. Gruber, M. Huber, A. Roth, 2009. TanDEM-X: Block adjustment of interferometric height models. Proceedings of ISPRS Hannover Workshop 2009 "High-Resolution Earth Imaging for Geospatial Information”, June 2 - 5, 2009, Hannover, Germany

[3] Huber, M., B. Wessel, D. Kosmann, A. Felbier, V. Schwieger, M. Habermeyer, A. Wendleder, and A. Roth, 2009. Ensuring globally the TanDEM-X height accuracy: Analysis of the reference data sets ICESat, SRTM, and KGPS-Tracks. Proceedings of IGARSS 2009, Cape Town, South Africa. 